\title{
KANSEI Based Clothing Fabric Image Retrieval
}

\author{
Yen-Wei Chen ${ }^{1,2}$, Shota Sobue ${ }^{2}$, and Xinyin Huang ${ }^{3}$ \\ ${ }^{1}$ Elect \& Inf. Eng. School, Central South Univ. of Forest and Tech., Changsha, China \\ ${ }^{2}$ Graduate School of Science and Engineering, Ritsumeikan University, Japan \\ ${ }^{3}$ School of Education, Soochow University, Suzhou, China
}

\begin{abstract}
KANSEI is a Japanese term which means psychological feeling or image of a product. KANSEI engineering refers to the translation of consumers' psychological feeling about a product into perceptual design elements. Recently KANSEI based image indexing or image retrieval have been done by using interactive genetic algorithms (IGA). In this paper, we propose a new technique for clothing fabric image retrieval based on KANSEI (impressions). We first learn the mapping function between the fabric image features and the KANSEI and then the images in the database are projected into the KANSEI space (psychological space). The retrieval is done in the psychological space by comparing the query impression with the projection of the images in database.
\end{abstract}

Keywords: Image retrieval, KANSEI, mapping function, image features, psychological space, impression, semantic differential (SD) method, neural network, principal component analysis.

\section{Introduction}

Recently a growing increase has been seen in digital images. Image retrieval has become an important issue for database management and computer vision. To date, many researches have been done for image retrieval. They can be divided into two groups: text-based image retrieval and image contents based image retrieval [1]. The text-based image retrieval [2] was started from the late of 1970's. In text-based image retrieval system, some keywords should be first manually annotated to each image in the database. The drawback of text-based image retrieval is that the vast amount of labor required in manual image annotation and the annotation impreciseness due to the subjectivity of human perception. In the early of 1990's, contents-based image retrieval was proposed to overcome the above problems. In contents-based image retrieval, images are indexed by their own visual content, such as color and texture [1, 3-7]. Both text-based and contents-based methods lack the capability of utilize human intuition and KANSEI (impression).

KANSEI is a Japanese term which means psychological feeling or image of a product. KANSEI engineering refers to the translation of consumers' psychological feeling about a product into perceptual design elements [8]. Recently KANSEI based image indexing or image retrieval systems have been done by using interactive genetic algorithms (IGA) [9,10]. In IGA-based image retrieval systems, the retrieval results are evaluated by users and the retrieval process is repeated until the user is 
satisfied. The IGA-based image retrieval system could retrieval the images based on human intuition or KANSEI (impression), but it is time and labor consuming method. In this paper, we propose a new technique for clothing fabric image retrieval based on KANSEI (impressions). We first learn the mapping function between the fabric image features and the human KNASEI factors. In our previous studies, we have significantly estimated the mapping functions from the image feature space to the KANSEI space for four groups with different ages [11]. We use the semantic differential (SD) method to extract the KANSEI factors (impressions) such as bright, warm from human while they viewing an fabric image. A neural network is used to learn the mapping functions from the image feature space to human KANSEI factor space (psychological space) and then the images in the database are projected into the psychological space. The retrieval is done in the psychological space by comparing the query impression with the projection of the fabric images in the database.

\section{Mapping Functions}

In order to make a quantitative study on the relationship between the image features and KANSEI factors, we construct an image feature space and a KANSEI factor space (psychological space) as shown in Fig.1.

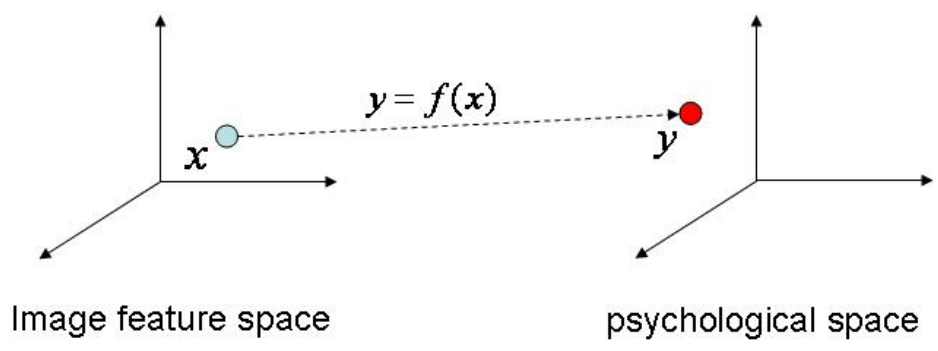

Fig. 1. Mapping Functions from image feature space into psychological space

One fabric image has one point in the image feature space and has a corresponding point in the KANSEI factor space, which is an impression (KANSEI) of the subject to the image, just like a projection of the image feature to the psychological space. The relationship between the image features and the KANSEI can be described by the mapping function from the image feature space to the psychological space. The input of the mapping function is the image features and the output of the function is the KANSEI factors (impressions). The mapping functions (relationships) can be learned by finding the corresponding points in the image feature space and psychological space.

\section{Psychological Features (Impressions)}

In order to find a corresponding point in psychological space, we use the semantic differential (SD) method to extract the KANSEI factors (impressions) such as bright, warm from 8 adults while they viewing an image (material). In this research, we use 
Table 1. 23 pairs of adjectives

\begin{tabular}{|ll|}
\hline 1. & strange - familiar \\
\hline 2. & unique - usual \\
\hline 3. & bright - dark \\
\hline 4. & Interesting - uninteresting \\
\hline 5. & scary - not scary \\
\hline 6. & pretty - ugly \\
\hline 7. & western - eastern \\
\hline 8. gorgeous - quiet \\
\hline 9. natural - artificially \\
\hline 10. clear - indistinct \\
\hline 11. fine - rough \\
\hline 12. adult - childlike \\
\hline 13. refreshing - messy \\
\hline 14. gentle - indifferent \\
\hline 15. deep - faint \\
\hline 16. regular - irregular \\
\hline 17. modern - classical \\
\hline 18. warm - cool \\
\hline 19. transparent - opaque \\
\hline 20. simple - complex \\
\hline 21. jaunty - placid \\
\hline 22. manly - womanly \\
\hline 23. like - dislike \\
\hline
\end{tabular}
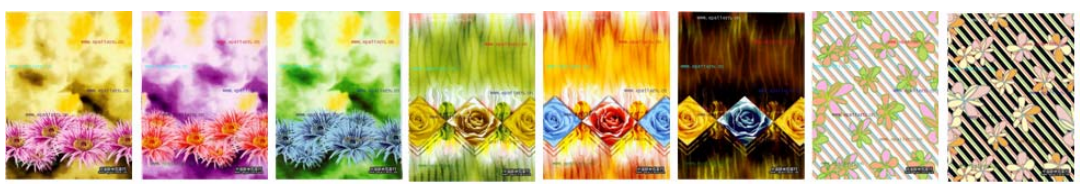

Fig. 2. Examples of clothing fabric images for training

words (adjective pairs) to measure the KANSEI. By careful selections, we chose 23 pairs of adjectives, which are shown in Table 1, as measures of KANSEI. We chose 168 clothing fabric images [12] for learning mapping functions and validations. Some examples are shown in Fig.2. We asked 8 college students to rate the impression with 23 pairs of adjectives in 7 -level scales $(-3,-2,-1,0,1,2,3)$.

\section{Image Features}

Low-level visual features such as color, texture and shape information in an image are used as image features. 

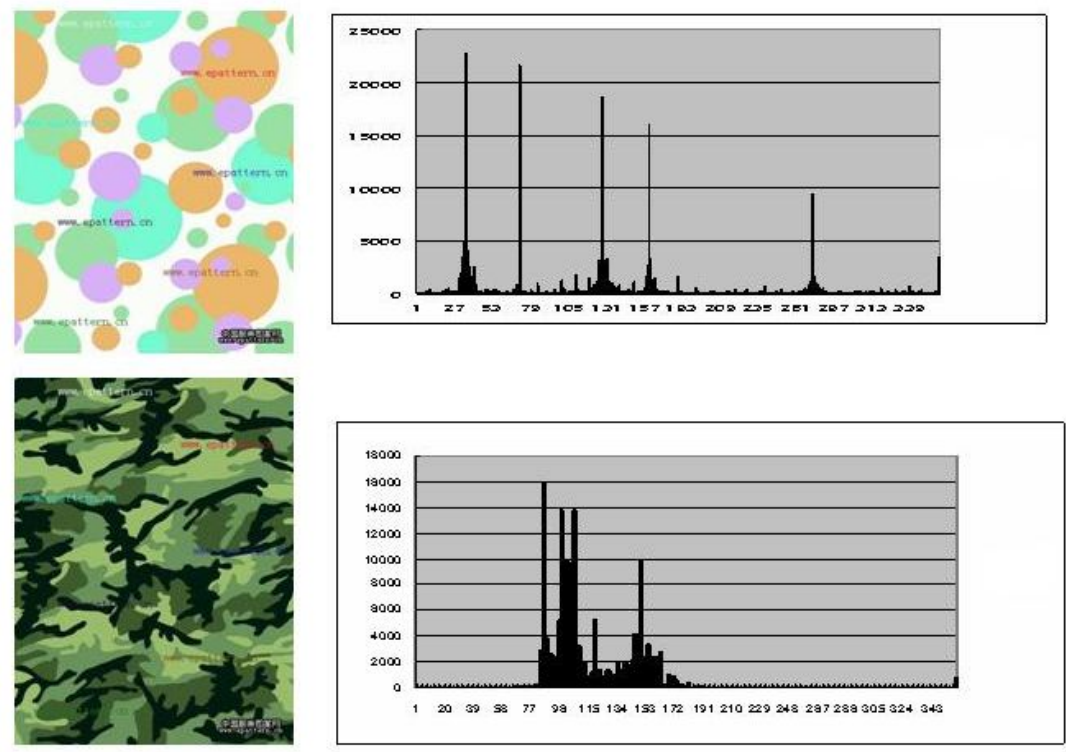

Fig. 3. Typical color fabric images and their color features

\subsection{Color Features}

Several color features have been proposed to represent color compositions of images [13]. Color histograms are widely used to capture the color information in an image [14]. They are easy to compute and tend to be robust against small changes of camera viewpoints. We first transform the color image from the RGB space to the HSV space and the Hue value which is used as color features is quantized into 360 bins. The gray levels ( $\mathrm{R}=\mathrm{G}=\mathrm{B}$ ) are represented by four bins. So the dimension of color feature vectors is 364 . Two typical color images and their color feature vectors are shown in Fig. 3. In order to find an efficient representation of color features, we use principal component analysis (PCA) to reduce the dimension of the color feature space. The 364-dimentional color space can reduced into 30-dimention, while maintaining $90 \%$ of information.

\subsection{Texture Features}

The texture features are represented by the use of Fourier transform power spectrum $\mathrm{P}(r, \theta)$, which express a periodic pattern of image in polar coordinate, where $r$ is the amplitude of the frequency and $\theta$ is the direction angle of the frequency. Following two features with dimensions of 50 and 180, respectively, are used for texture representation:

$$
\begin{aligned}
& p(r)=\sum_{\theta=0}^{\pi} P(r, \theta) \\
& q(\theta)=\sum_{r=0}^{w / 2} P(r, \theta)
\end{aligned}
$$



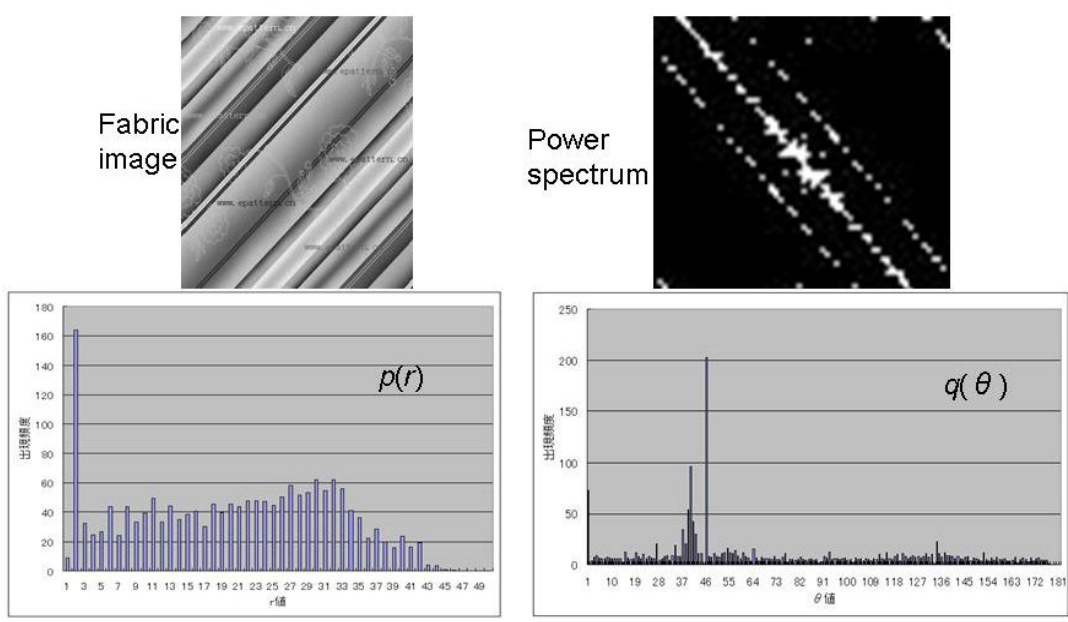

Fig. 4. A typical fabric image and its texture features

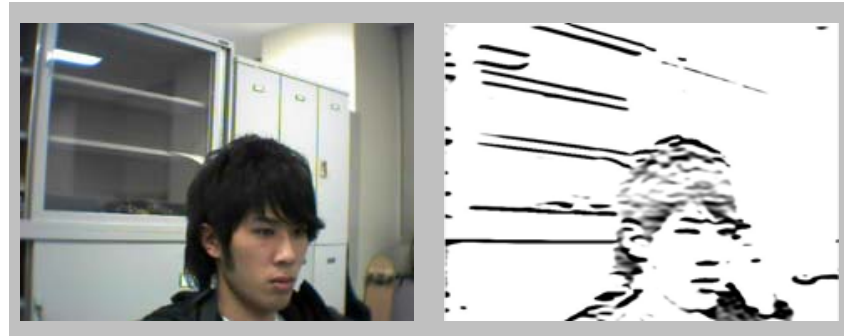

Fig. 5. A typical image and its Gabor filtered image $(\alpha=\pi / 2)$

The typical fabric image and its features are shown in Fig.4. The dimension of the texture feature vector, which is composed of $p(r)$ and $q(\theta)$, is 230 and it can reduced into only 2 by PCA, while maintaining $70 \%$ of information.

\subsection{Shape Features}

We use Gabor filters to extract shape features. A directional Gabor filter can be expressed as:

$$
\begin{aligned}
& F(x, y)=e^{-\pi\left(x^{2} a^{2}+y^{2} b^{2}\right)} \cos (u x+v y) \\
& u=f \cos \alpha, v=f \sin \alpha \\
& f: \text { frequency }
\end{aligned}
$$

where $\alpha$ is the direction of the filter. Four directional Gabor filters with angles of 0 , $\pi / 4, \pi / 2, \pi 3 / 4$, respectively, are used for shape feature extractions. Each filtered image is divided into $10 \times 10$ sub-images and the mean value of each sub-image is used as 
shape features. Thus the dimension of the shape feature vector is 400 and it can reduced to 15 by PCA, while maintaining $80 \%$ of information. A typical image and its Gabor filtered image $(\alpha=\pi / 2)$ are shown in Fig. 5. It can be seen that the horizontal features are extracted.

\section{Learning and Validation}

In this paper, we use a neural network as a model of mapping function as shown in Fig.6. The neural network can be used to approximate any nonlinear functions. The neural network or the mapping function can be learned by finding the corresponding points in the image feature space and psychological space. The input of the neural network is the image features and the output is the corresponding impression. The number of input neurons is $47(30+2+15)$, the number of the output neurons is 23 (the number of the pairs of adjectives) and the range of output neurons is [-3, 3$]$. The number of the neurons in middle layer is 67.

We choose 165 images as training images to train the neural network. Once the neural network is trained, we use remained 3 images, which are not included in the training images, as test images for validations. We compared the outputs (estimated impressions) of the test images with real impressions obtained by SD method and calculate the mean squared error (MSE) between the estimated impression and real impression. The experimental process is shown in Fig.6(b).

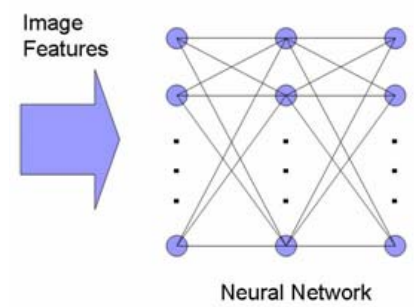

(a)

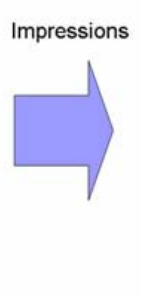

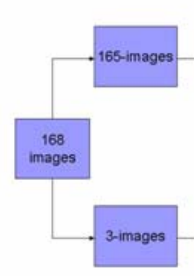

- Feature

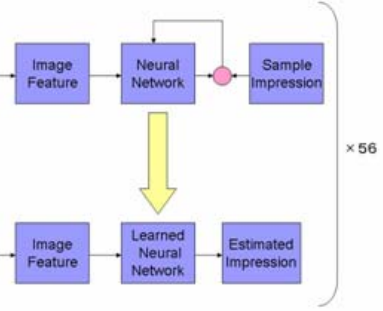

(b)

Fig. 6. (a) 3-layer neural network; (b) experiment process for training and validation

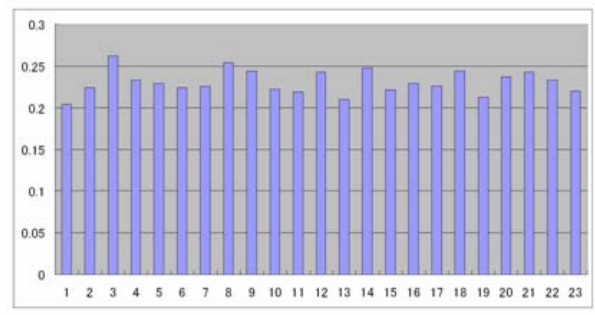

(a)

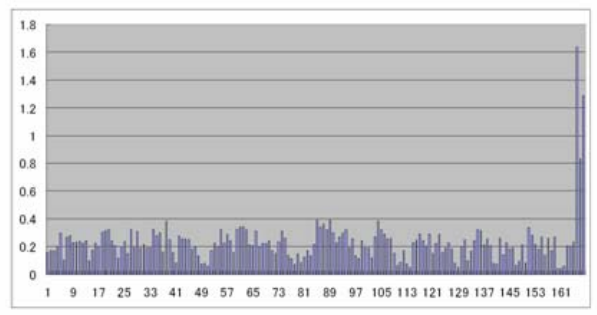

(b)

Fig. 7. (a) Averaged MSE of each impression; (b) Overall MSE of each image 
The experiments are repeated 56 times with different training and test images. Averaged MSE of each impression is shown in Fig.7(a). Since the value of the impression is $-3 \sim 3$, the estimation error is less than $10 \%$. The overall MSE of each image is shown in Fig.7(b).

\section{KANSEI Based Image Retrieval}

As an application, we developed a KANSEI based clothing fabric image retrieval system. The flowchart of the system is shown in Fig.8.

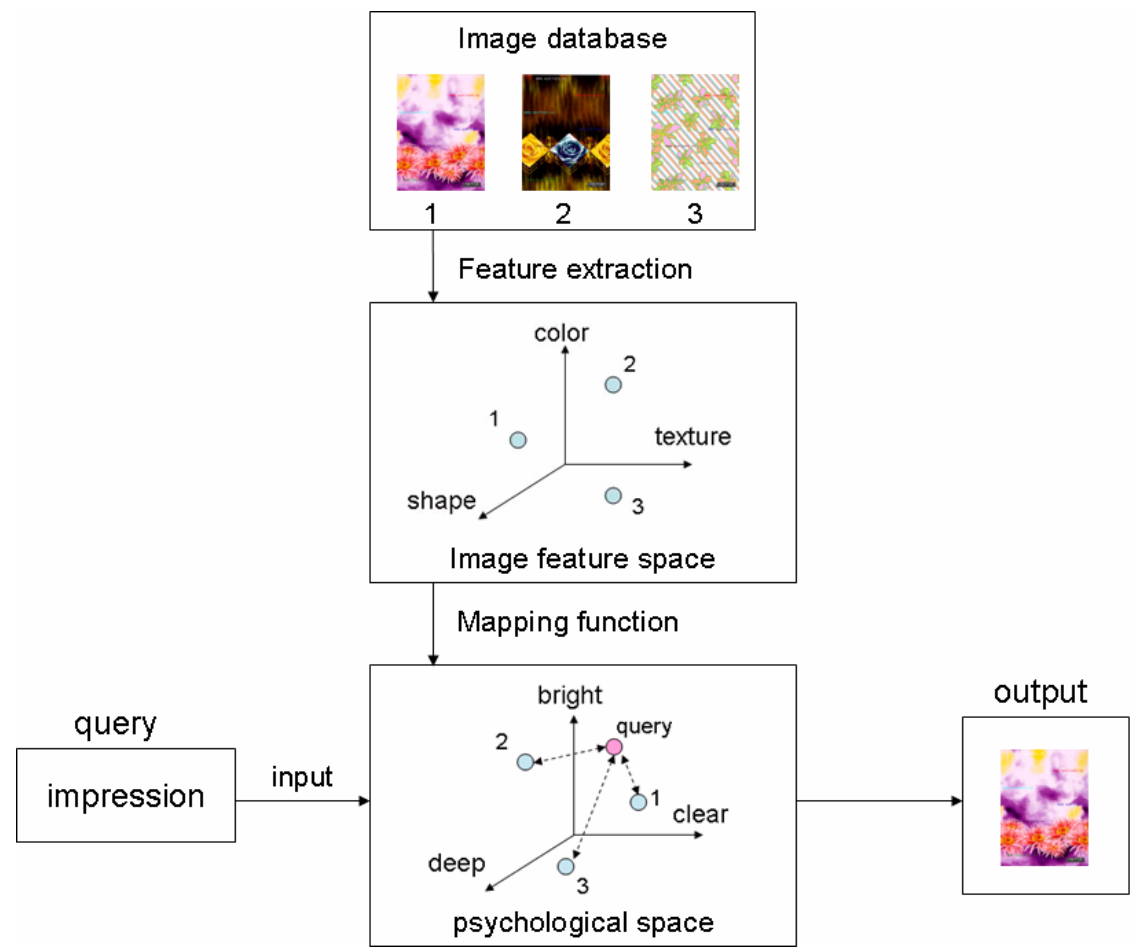

Fig. 8. Flowchart of KANSEI based image retrieval system

The input query is KANSEI words (impressions). The image features (color, texture and shape features) of fabric images in the database are first extracted by use the methods, which are shown in Sec.4) and then the image feature vector is projected or transformed into the psychological space (impression vector) by the mapping function (trained neural network). We calculated the Euclid distance between the query impression vector and the each transformed fabric image impression vector. The image with minimum distance is retrieved as an output. The examples of the retrieval results are shown in Fig.9(a) and 9(b). The retrieval results for query impressions of "bright", 

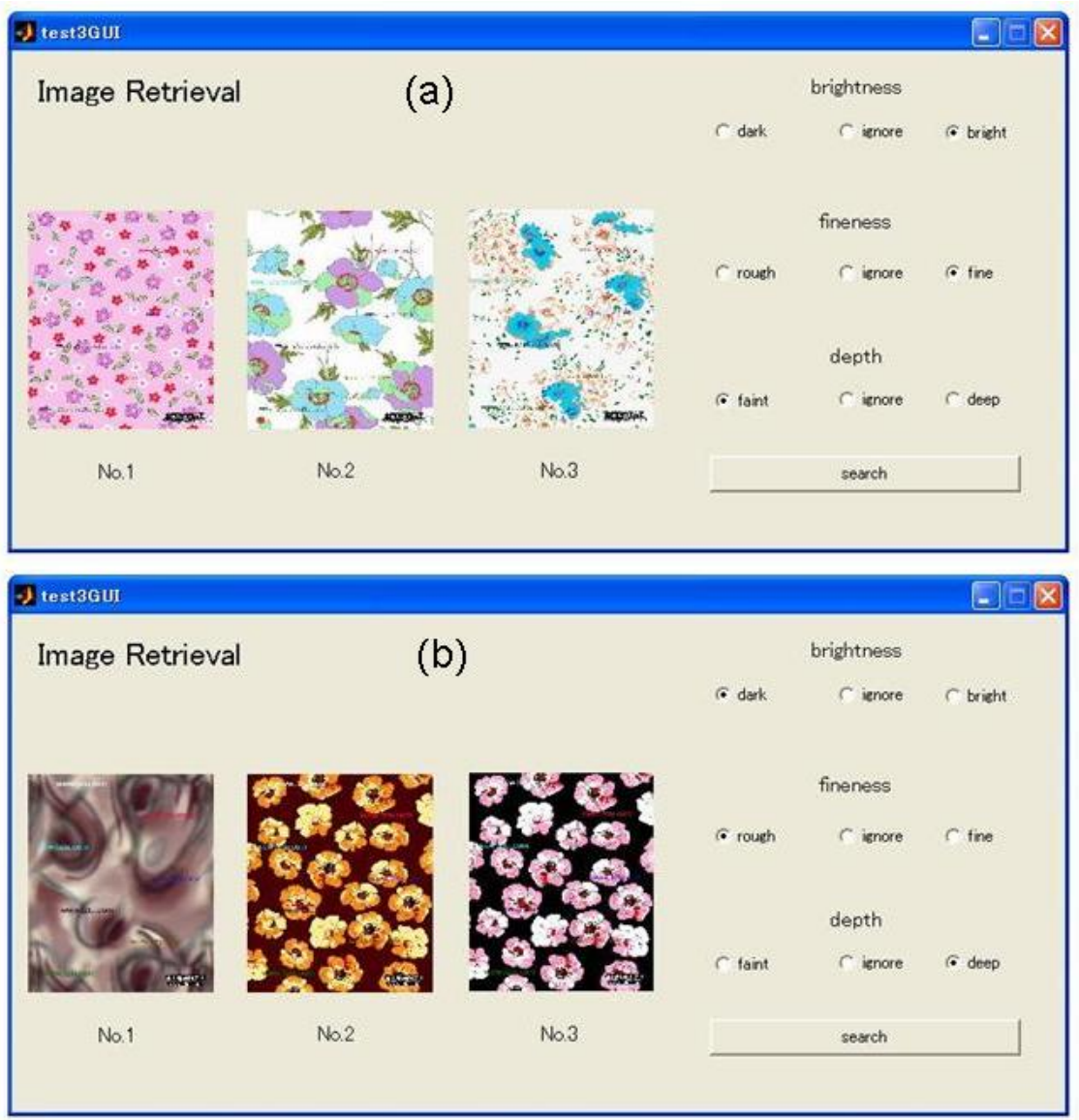

Fig. 9. Retrieval results for query impressions. (a) "bright", "fine", and "faint"; (b) "dark", "rough" and "deep".

"fine", and "faint" are shown in Fig.9(a) and the retrieval results for the query impression of "dark", "rough" and "deep" are shown in Fig.9(b). It can be seen that the retrieval images are marched with the query impressions. In order to make a quantitative evaluation, the levels of retrieval images, which are measured by the SD method (Sec.3), are listed in Table 2. "Bright" has a positive value and the highest level is +3 , while "dark" has a negative value and the highest level is -3 . As shown in Table 1, the retrieval images are also evaluated by human as "bright" and "dark", respectively.

Table 2. Human evaluated levels of the retrieval images

\begin{tabular}{|c|c|c|c|}
\hline Query & No.1 & No.2 & No.3 \\
\hline Bright & 2.17 & 1.67 & 1.67 \\
\hline Dark & -1.83 & -0.10 & -0.13 \\
\hline
\end{tabular}




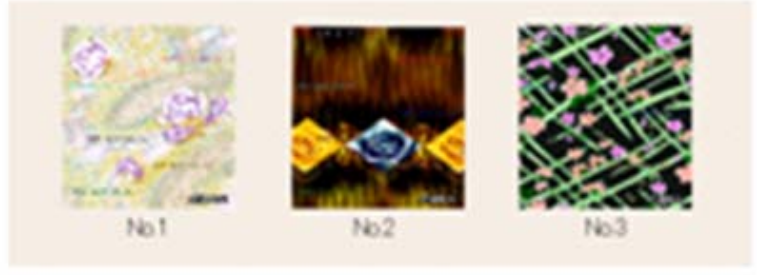

1st generation

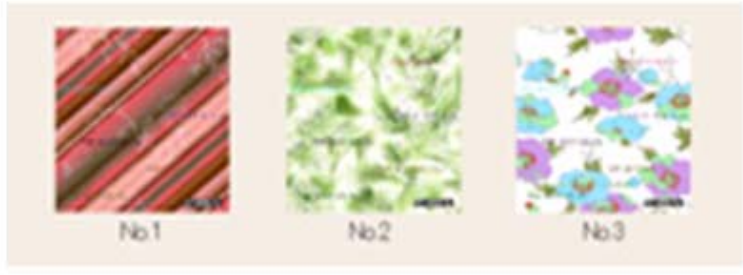

\section{5th generation}

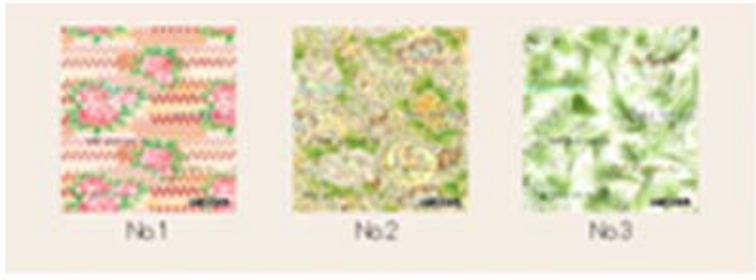

\section{0th generation}

Fig. 10. Retrieval images for a query of "bright" by conventional IGA

The retrieval images for a query of "bright" by conventional IGA [15] are shown in Fig.10. It can be seen that in order to obtain a satisfied result, the retrieval process should be repeated 10 times (10 generations). It is time and labor consuming.

\section{Conclusion}

We proposed a novel approach to learn the mapping function from the image features space to the human KANSEI space (psychological space) using a multi layer neural network. Experimental results show that for a given image, the KANSEI (impressions) estimation error is less than $10 \%$. As an application, we also developed a KANSEI based clothing fabric image retrieval system. Significant positive results have been obtained. We can retrieve the desired clothing fabric images from the database by using only some KANSEI words (impression words). 


\section{Acknowledgements}

This work is supported in part by the "Open Research Center" Project for Private Universities: matching fund subsidy from Japanese Ministry of Education, Culture, Sports, Science, and Technology.

\section{References}

1. Rui, Y., Huang, T.S.: Image retrieval: Current techniques, promising directions, and open Issues. Journal of Visual Communication and Image Representation 10, 39-62 (1999)

2. Tamura, H., Yokoya, N.: Image database systems: A survey. Pattern Recognition 17(1) (1984)

3. Niblack, W., et al.: The QBIC project: Querying images by content using color, texture, and shape. In: Storage and Retrieval for Image and Video Databases, pp. 173-187. SPIE (1993)

4. Hirata, K., Kato, T.: Query by visual example: Content based image retrieval. In: Advances in Database Technology, EDBT 1992, pp. 56-61 (1992)

5. Pentland, A., Picard, R., Sclaroff, S.: Photobook: Content-based manipulation of image databases. In: SPIE Storage and Retrieval for Image and Video Databases. SPIE (1994)

6. Zeng, X.-Y., Chen, Y.-W., Nakao, Z., Cheng, J., Lu, H.: Independent Component Analysis for Color Indexing. IEICE Trans. Information \& Systems E87-D, 997-1003 (2004)

7. Han, X.-H., Chen, Y.-W., Sukegawa, T.: A supervised nonlinear neighbourhood embedding of color histogram for image indexing. In: Proc. of 15th IEEE International Conference of Image Processing (ICIP 2008), USA, pp. 949-953 (2008)

8. Grimsæth, K.: Linking emotions and product features. KANSEI Engineering, 1-45 (2005)

9. Cho, S.B., Lee, J.Y.: A human-oriented image retrieval system using iterative genetic algorithm. IEEE Trans. Systems, Man and Cybernetics, Part A 32, 452-458 (2002)

10. Takagi, H., Noda, T., Cho, S.: Psychological Space to Hold Impression Among Media in Common for Media Database Retrieval System. In: Proc. of IEEE International Conference on System, Man, and Cybernetics (SMC 1999), Tokyo, Japan, vol. VI (1999)

11. Huang, X., Sobue, S., Kanda, T., Chen, Y.-W.: Linking KANSAI and image features by multi-layer neural networks. In: Apolloni, B., Howlett, R.J., Jain, L. (eds.) KES 2007, Part II. LNCS (LNAI), vol. 4693, pp. 318-325. Springer, Heidelberg (2007)

12. http: //www. epattern.cn/

13. Sticker, M.A., Orengo, M.: Similarity of color images. In: Proc. SPIE Storage Retrieval Still Image Video Database, pp. 381-392 (1996)

14. Deng, Y., Manjunath, B.S.: An efficient color representation for image retrieval. IEEE Trans. Image Processing 10, 140-147 (2001)

15. Sobue, S.: KANSEI based image retrieval by SVM and IGA. Ritsumeikan University Master's Thesis (2008) 